\title{
Morphological differentiation and clavulanic acid formation are affected in a Streptomyces clavuligerus adpA-deleted mutant
}

Correspondence

Paloma Liras

paloma.liras@unileon.es

Received 29 October 2009

Revised 3 May 2010

Accepted 5 May 2010

\author{
M. Teresa López-García, ${ }^{1,2}$ Irene Santamarta ${ }^{1,2}$ and Paloma Liras ${ }^{1,2}$ \\ ${ }^{1}$ Área de Microbiología, Facultad de Ciencias Biológicas y Ambientales, Universidad de León, \\ 24071 León, Spain \\ ${ }^{2}$ Instituto de Biotecnología, INBIOTEC, Parque Científico de León, Avda. Real nº 1, 24006 León, \\ Spain
}

\begin{abstract}
The TTA codon-containing adpA gene of Streptomyces clavuligerus, located upstream of ornA, is in a DNA region syntenous with the homologous region of other Streptomyces genomes. Deletion of $\operatorname{adp} A$ results in a medium-dependent sparse aerial mycelium formation and lack of sporulation. Clavulanic acid formation in this mutant decreases to about $10 \%$ of the wild-type level depending on the medium, whereas its production is strongly stimulated by increasing the $\operatorname{adp} A$ copy number. Quantitative transcriptional analysis indicates that expression of the clavulanic acid regulatory genes ccaR and claR decreases seven- and fourfold, respectively, in the $\Delta a d p A$ mutant, resulting in a large decrease in expression of genes encoding biosynthesis enzymes for the early steps of clavulanic acid formation and a smaller decrease in the expression of genes for the late steps of the pathway. An ARE box, 5'-TCTCATGGAGACATAGCGGGGCATGC-3', is present upstream of adpA and efficiently binds $S$. clavuligerus Brp protein, as shown by electrophoretic mobility shift assay (EMSA) analysis. The transcription level of adpA is higher in the absence of Brp, as shown in $S$. clavuligerus $\Delta b r p$, suggesting a connection between $\operatorname{adp} A$ expression and the $\gamma$-butyrolactone system in $S$. clavuligerus.
\end{abstract}

\section{INTRODUCTION}

Streptomycetes are of particular interest as producers of a variety of well-known enzymes and secondary metabolites of commercial value, such as antibiotics, anti-tumour agents, immunosuppressors and enzyme inhibitors. Secondary metabolite production is specifically regulated at several levels of control, involving a regulatory network with different degrees of complexity. In some cases the regulatory network affects the production of one of the antibiotics produced by the strain, while in other cases the production of several antibiotics along with morphological development is affected.

A well-characterized regulatory cascade of Streptomyces griseus controls morphological and biochemical differentiation to secondary metabolism (Ohnishi et al., 2005). Afactor, a microbial hormone active at about $100 \mathrm{nM}$ concentration, binds the cytoplasmic receptor protein ArpA and releases it from specific DNA sequences named ARE boxes. An ARE box, located upstream of the pleiotropic regulator gene $a d p A$, is responsible for the negative control exerted by ArpA on adpA expression. Derepression of adpA results in the formation of AdpA, a

Abbreviation: EMSA, electrophoretic mobility shift assay. member of the AraC/XylS-type regulatory protein subfamily, which activates a gene regulon. This includes genes $(a d s A, s s g A)$ for morphogenesis and spore formation (Yamazaki et al., 2000, 2003), and for chymotrypsin, trypsin, serine proteases and metalloendopeptidases (sprA, $B, D, T, U$; sgmA) (Kato et al., 2002, 2005; Tomono et al., 2005b) with multiple functions in cell development. In addition, in S. griseus, AdpA activates expression of pathway-specific regulatory genes $(s t r R, g r i R)$, triggering streptomycin and grixazone biosynthesis and resistance (Higashi et al., 2007; Tomono et al., 2005a). Genes belonging to the AdpA regulon possess, upstream of their promoters, specific AdpA-binding sequences, such as $5^{\prime}$ TGGCSNGWWY-3' in S. griseus, where $\mathrm{S}=\mathrm{G} / \mathrm{C}, \mathrm{W}=\mathrm{A} / \mathrm{T}$, $\mathrm{Y}=\mathrm{T} / \mathrm{C}$ and $\mathrm{N}$ is any nucleotide (Ohnishi et al., 2005; Yamazaki et al., 2004).

In the model micro-organism Streptomyces coelicolor, $a d p A_{c}$ (previously bldH) is not essential for undecylprodigiosin production but is required for actinorhodin formation (Takano et al., 2003). In addition, $a d p A_{c}$ is normally expressed in butyrolactone non-producing $s c b A$ and $s c b R$-disrupted mutants (ScbR being orthologous to ArpA and Brp in S. griseus and Streptomyces clavuligerus, respectively). Therefore, ArpA control over adpA expression appears to be different in S. coelicolor. The lack of 
antibiotic production and sporulation by adpA-negative mutants of Streptomyces ansochromogenes has been related to the presence of multiple AdpA-binding sites in the upstream region of $\operatorname{san} G$, a gene encoding the specific activator protein for nikkomycin production (Pan et al., 2009).

All adpA genes contain a TTA codon translated in Streptomyces by the rare bldA-encoded tRNA ${ }^{\text {leu }}$ (Chater \& Chandra, 2008; Lawlor et al., 1987). TTA codons are present in many antibiotic-specific regulators, which explains the non-producing phenotype of $S$. coelicolor bldA mutants (Fernández-Moreno et al., 1991; White \& Bibb, 1997). The inability to translate adpA also explains the bald phenotype of the $S$. coelicolor bldA mutant (Nguyen et al., 2003; Takano et al., 2003), in which aerial mycelium formation is restored by complementation with a TTA-free adpA gene (Nguyen et al., 2003).

In S. clavuligerus, cephamycin $\mathrm{C}$ and clavulanic acid production are activated by the CcaR regulator (PérezLlarena et al., 1997). CcaR formation is modulated by regulatory proteins such as Brp, a butyrolactone receptor protein, and AreB, an IclR-like protein that connects primary and secondary metabolism (Santamarta et al.,
$2005,2007)$. In addition, clavulanic acid production is specifically activated by ClaR, a regulator under CcaR control (Paradkar \& Jensen, 1998; Pérez-Redondo et al., 1999).

In this paper we report that $S$. clavuligerus AdpA is part of a regulatory cascade that controls antibiotic production and study whether its involvement in biochemical and morphological differentiation is similar to that found in $S$. griseus.

\section{METHODS}

Bacterial strains, plasmids and culture conditions. The bacterial strains and plasmids used in this study are listed in Table 1. Escherichia coli strain DH5 $\alpha$ was maintained on Luria broth agar plates (Sambrook et al., 1989) and grown in Luria broth liquid medium at $37{ }^{\circ} \mathrm{C}$. Cultures of plasmid-bearing cells were supplemented with ampicillin $\left(50 \mu \mathrm{g} \mathrm{ml}^{-1}\right)$, chloramphenicol $\left(25 \mu \mathrm{g} \mathrm{ml}^{-1}\right)$, kanamycin $\left(25 \mu \mathrm{g} \mathrm{ml}^{-1}\right)$ or apramycin $\left(50 \mu \mathrm{g} \mathrm{ml}^{-1}\right)$ as appropriate. E. coli Ess22-35 and Klebsiella pneumoniae ATCC 29665 were used in cephamycin $\mathrm{C}$ and clavulanic acid bioassays, respectively (Liras \& Martín, 2005).

S. clavuligerus ATCC 27064 and S. clavuligerus mutant strains were maintained on $2 \%$ agar TSB medium $\left(30 \mathrm{~g}^{-1}\right.$ tryptic casein soy

Table 1. Bacterial strains and plasmids used in this study

\begin{tabular}{|c|c|c|}
\hline Strain or plasmid & Relevant features* & Reference or source \\
\hline S. clavuligerus ATCC 27064 & Wild-type; cephamycin $\mathrm{C}$ and clavulanic acid producer & $\mathrm{ATCC} \dagger$ \\
\hline S. clavuligerus $\Delta b l d A$ & bldA-deleted mutant & Trepanier et al. (2002) \\
\hline S. clavuligerus $\triangle a d p A[\mathrm{pCPA} 2]$ & $\begin{array}{l}\text { adpA-deleted mutant complemented with } a d p A \text { and its own } \\
\text { promoter region }\end{array}$ & This study \\
\hline S. clavuligerus pIJ699 & Wild-type strain transformed with the multi-copy vector pIJ699 & This study \\
\hline S. clavuligerus $\mathrm{pIJ} a d p A$ & Wild-type strain transformed with pIJadpA & This study \\
\hline E. coli ET12567 & Methylation-deficient & Kieser et al. (2000) \\
\hline E. coli ET12567(pUZ8002) & Methylation-deficient; transfer functions from pUZ8002 & Kieser et al. (2000) \\
\hline E. coli Ess $22-31$ & $\beta$-Lactam antibiotic-supersensitive & Romero et al. (1984) \\
\hline E. coli $\mathrm{DH} 5 \alpha$ & General cloning host & Stratagene \\
\hline \multicolumn{3}{|l|}{ Plasmids } \\
\hline pTC192-Km & $\begin{array}{l}\text { pUC19-derived vector containing Kan-resistance gene (aphII) } \\
\text { from Tn } 5 \text { transposon }\end{array}$ & Rodríguez-García et al. (2006) \\
\hline pIJ773 & Apr $^{\mathrm{r}}$ cassette in $\mathrm{pIJ} 699$ & Gust et al. (2003) \\
\hline pIJ699 & Multi-copy Streptomyces vector containing Thio-resistance gene & Kieser \& Melton (1988) \\
\hline pMS83 & Integrative vector used for adpA-deleted mutant complementation. & M. Smith, University of Aberdeen \\
\hline $\mathrm{pIJ} a d p A$ & $a d p A$ and its promoter region in pIJ699 & This study \\
\hline $\mathrm{p} \Delta a d p A$ & $a d p A:: a c c$-inactivation construct & This study \\
\hline $\mathrm{pP} a d p A$ & $a d p A$ and its promoter region (448 bp) in pBluescript II $\mathrm{KS}(+)$ & This study \\
\hline рСРА2 & adpA genetic complementation vector & This study \\
\hline
\end{tabular}

*Amp, ampicillin; Apr, apramycin; Kan, kanamycin; Thio, thiostrepton.

$\dagger$ ATCC, American Type Culture Collection. 
broth) at $28{ }^{\circ} \mathrm{C}$. ME medium, containing (in $\mathrm{g}^{-1}$ ) MOPS (21), glucose (5), yeast extract (0.5), meat extract (0.5), caseine peptone (1), agar (20), pH 7.0 (Sánchez \& Braña, 1996), or TBO medium, containing (in $\mathrm{g}^{-1}$ ) tomato paste (20), oat flakes (20), agar (20), pH 6.5 (Higgens et al., 1974), were used to test the morphological differentiation and spore formation ability of Streptomyces mutant strains when compared with the wild-type strain. For antibiotic production studies and transcriptional analysis, SA defined or TSB complex medium was used (Lorenzana et al., 2004; Paradkar \& Jensen, 1998). Strains were grown in $500 \mathrm{ml}$ baffled flasks containing $100 \mathrm{ml}$ TSB medium at $28{ }^{\circ} \mathrm{C}$ and 220 r.p.m. for 24 h. Five millilitres of the culture were harvested, and the mycelium was washed with $0.9 \% \mathrm{NaCl}$ and used to inoculate SA medium. Triplicate cultures were incubated at $28{ }^{\circ} \mathrm{C}$ and 250 r.p.m. The growth rate was determined by measuring the DNA concentration using the diphenylamine reaction (Burton, 1968).

Nucleic acid manipulations. General DNA manipulations were performed using standard techniques (Sambrook et al., 1989). Streptomyces genomic and plasmid DNA preparations, S. clavuligerus conjugation with E. coli ET1257/pUZ8002 as donor strain, and Streptomyces protoplast transformation were done following standard methods (Kieser et al., 2000). Nucleic acid hybridizations were performed following the DIG system protocol (Roche), and colorimetric detection was carried out with nitro blue tetrazolium (NBT) and 5-bromo-1-chloro-3-indolyl phosphate (BCIP).

RNA samples from S. clavuligerus strains were prepared using RNeasy Mini spin columns (Qiagen), as previously described by Santamarta et al. (2005), and treated with DNase I (Qiagen) and Turbo DNase (Ambion) to eliminate chromosomal DNA contamination. PCR and RT-PCR were performed in a T-gradient (Biometra) thermocycler. Plasmids and oligonucleotides used in this work are shown in Tables 1 and 2, respectively.

\section{Plasmid construction}

Construction of $\mathrm{p} \Delta a d p A$ for $\operatorname{adp} A$ deletion. DNA fragments upstream (UP-adpA, $1983 \mathrm{bp}$ ) and downstream (D-adpA, $2267 \mathrm{bp}$ ) of $a d p A$ were amplified by PCR using oligonucleotides UpadpA-O1 and UpadpA-O2, and DWadpA-O1 and DWadpA-O1, respectively. Once sequenced, fragment UP-adpA was subcloned into the EcoRV site of pBluescriptII KS to form pUP-adpA. The apramycin-resistance cassette from pIJ773, containing the origin for conjugation (oriT) and the apramycin-resistance gene, was ligated into a filled blunt HindIII site of pUP-adpA, to obtain the vector $\mathrm{pU}$ : aac; the PCR-amplified D$a d p A$ fragment was subcloned into a filled blunt ClaI site of vector $\mathrm{pU}$ : aac to give plasmid $\mathrm{pU}: a c c: \mathrm{D}$ in such a way that the apramycinresistance gene was expressed divergently from the ornA gene. After SpeI linearization, $\mathrm{pU}$ : acc:D was ligated to the $1.4 \mathrm{~kb} X b a \mathrm{I}$ DNA fragment containing the aphII gene for kanamycin resistance, isolated from plasmid pTC192-Km, leading to plasmid $\mathrm{p} \Delta a d p A$. This plasmid was conjugated into $S$. clavuligerus, and apramycin-resistant transconjugants were subjected to sporulation on solid soy-mannitol medium in the absence of antibiotic and then plated onto antibioticsupplemented medium. Apramycin-resistant kanamycin-sensitive gene adpA-replacement mutants were confirmed by Southern hybridization.

Construction of pCPA2 to complement S. clavuligerus $\triangle a d p A$. The $a d p A$ gene with its own promoter (1580 bp) was PCR-amplified using oligonucleotides $\mathrm{P} a d p A-\mathrm{O} 1$ and $\mathrm{P} a d p A-\mathrm{O} 2$. The amplified fragment was subcloned into the EcoRV site of pBluescriptII KS, giving $\mathrm{pPadpA}$. PvuII digestion of pPadpA produced a $2034 \mathrm{bp}$ fragment that was ligated into the EcoRV site of pMS83, leading to pCPA2. Plasmid pMS83 derives from pMS82 and uses the integration site for Streptomyces phage ФBT1 (Gregory et al., 2003).
Construction of pJadpA to amplify the copy number of adpA. The adpA gene with its own promoter was amplified using oligonucleotides $\mathrm{Pad} A A-\mathrm{O} 1$ and $\mathrm{Pad} A A-\mathrm{O} 2$. The amplified and sequenced fragment was subcloned in the DraI site of the highcopy-number plasmid pIJ699, producing plasmid pIJadpA, used to overexpress adpA in S. clavuligerus.

Mobility shift assays. The $\mathrm{ARE}_{a d p A^{-c o n t a i n i n g ~ p r o b e ~ w a s ~ i s o l a t e d ~ a s ~}}$ a $448 \mathrm{bp} \mathrm{AvaI}$ fragment from plasmid pIJadpA. Once labelled with DIG-11-dUTP (DIG Gel Shift kit, 2nd generation, Roche) for chemiluminiscence detection it was applied to DNA-binding assays using pure $\mathrm{rBrp}$ protein $(0.5 \mu \mathrm{g}$ ) (Santamarta et al., 2005). Once electrophoretic mobility shift assays (EMSAs) had been performed (Santamarta et al., 2007), gels were transferred in $0.5 \times$ TBE buffer to Hybond-N + membranes (GE Healthcare) and developed for detection of DIG-11-dUTP-labelled fragments.

\section{Immunodetection of ApdA}

Generation of antibodies. A peptide corresponding to amino acids 385-399 of the AdpA sequence $\left(\mathrm{NH}_{2}\right.$-CAGHGRPSLPGQRSAP$\mathrm{COOH}$ ) was commercially synthesized (NeoMPS, Strasbourg, France). To raise polyclonal antibodies, the peptide $(3 \mathrm{mg})$ was resuspended in $1 \mathrm{ml}$ PBS ( $\mathrm{pH} \mathrm{7.5)}$ and thoroughly mixed with Freund's complete adjuvant. The solution was injected subcutaneously at multiple sites in New Zealand rabbits. The injections were repeated after 2 and 4 weeks using $1 \mathrm{mg}$ peptide only. A blood sample was taken 1 week after the final injection. After allowing the blood to clot at room temperature, the serum was collected by centrifugation and stored at $-20{ }^{\circ} \mathrm{C}$ until further use. To purify antibodies, the immunizing peptide was coupled to CNBr-activated Sepharose $4 \mathrm{~B}$ according to the supplier's instructions. The serum was passed through the affinity column and washed with PBS ( $\mathrm{pH} 7.5$ ). Antibodies were eluted by washing the column with $0.1 \mathrm{M}$ glycine $(\mathrm{pH} 3.0)$ and collected fractions $(1 \mathrm{ml})$ were immediately neutralized by the addition of $1 \mathrm{M}$ Tris and stored at $-20{ }^{\circ} \mathrm{C}$ until use.

Western-blotting assays. AdpA was immunodetected in $S$. clavuligerus cell-free protein extracts as follows. Mycelium from a $36 \mathrm{~h}$ culture was washed with and resuspended in lysis buffer $(10 \mathrm{mM}$ Tris/HCl, 1 mM EDTA, pH 7.5). After disruption by sonication, cell debris was removed by centrifugation at $4{ }^{\circ} \mathrm{C}$ and 14000 r.p.m. for $30 \mathrm{~min}$. Samples ( $5 \mu \mathrm{g}$ protein) were electrophoretically separated by $12 \%$ SDS-PAGE and blotted to a PVDF membrane (Immobilon-P, Millipore) for inmunodetection using an alkaline phosphataseconjugated anti-rabbit secondary antibody.

PCR, RT-PCR analysis and real-time RT-PCR. Total DNA of $S$, clavuligerus was used to amplify: (i) the complete adpA ORF (1206 bp) using oligonucleotides adpA-O1/adpA-O2; (ii) adpA downstream (2267 bp) and upstream (1983 bp) regions using DWadpA-O1/DWadpA-O2 and UpadpA-O1/UpadpA-O2, respectively; (iii) a fragment containing the $a d p A$ promoter region and ORF using the oligonucleotides PadpA-O1/adpA-O2. Every PCR $(20 \mu \mathrm{l})$ was performed as described by Kieser et al. (2000) and contained $300 \mathrm{ng}$ DNA template, $0.5 \mathrm{mM}$ each oligonucleotide, $28 \mathrm{mM}$ each dGTP and dCTP, $12 \mathrm{mM}$ each dATP and dTTP, $1 \mathrm{mM}$ $\mathrm{MgCl}_{2}, 5 \%$ DMSO and $0.8 \mathrm{U}$ Platinum Pfx DNA Polymerase (Invitrogen). With small variations of annealing temperature, the PCR program was as follows: after the first step at $95{ }^{\circ} \mathrm{C}$ for $30 \mathrm{~s}$, the annealing temperature was reduced in a touch-down of $1{ }^{\circ} \mathrm{C}$ from 65 to $58{ }^{\circ} \mathrm{C}$ in one cycle, and an annealing temperature of $58{ }^{\circ} \mathrm{C}$ was used in the next 25 cycles with an extension step of 2 min at $72{ }^{\circ} \mathrm{C}$. The PCR products were confirmed for size and purity by agarose gel electrophoresis, isolated from the gel using the Qiagen II DNA Cleanup System (Qiagen) and sequenced. 
Table 2. Oligonucleotide pairs used in this study

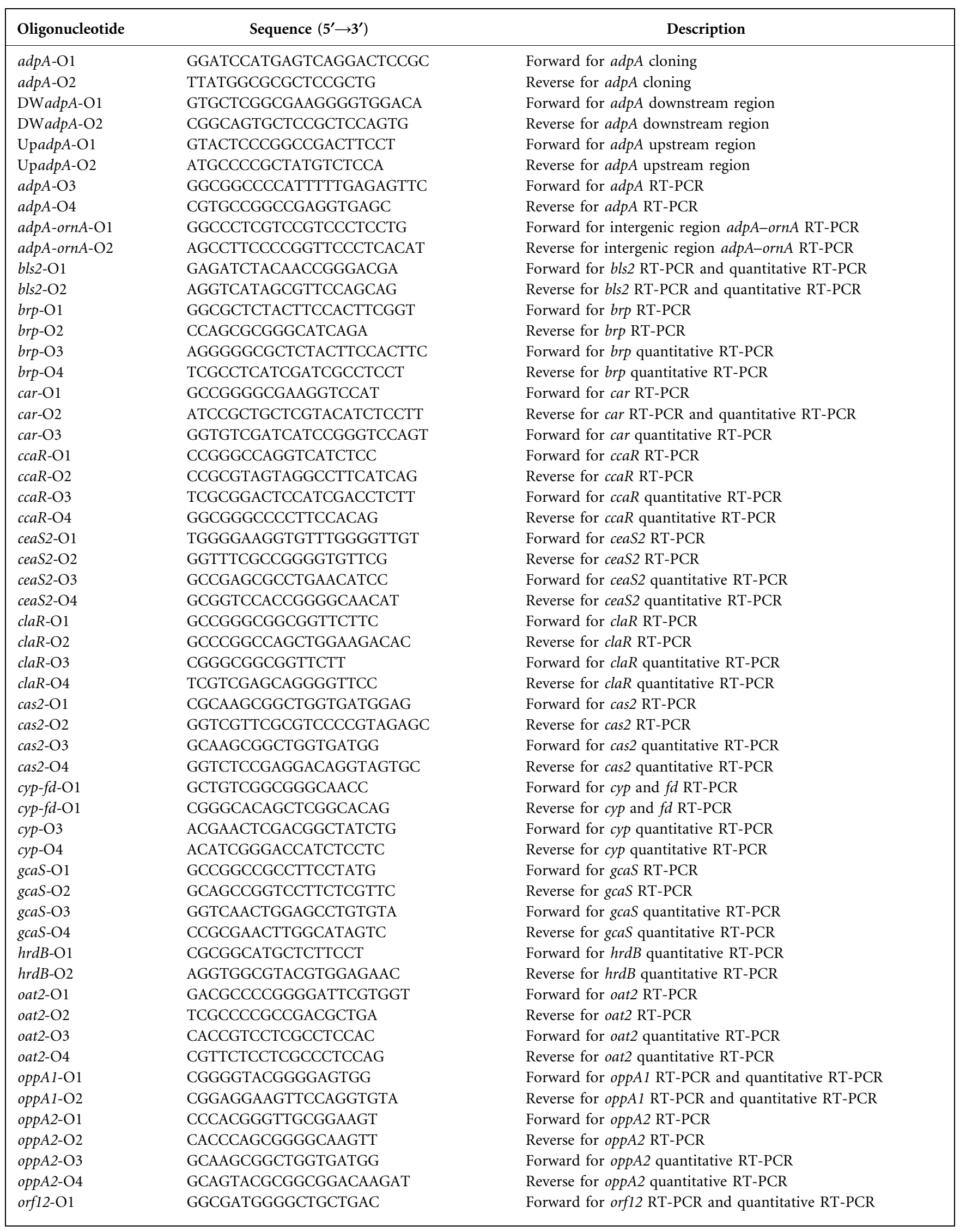


Table 2. cont.

\begin{tabular}{|lll|}
\hline Oligonucleotide & \multicolumn{1}{c|}{ Sequence $\left(\mathbf{5}^{\prime} \rightarrow \mathbf{3}^{\prime}\right)$} & \multicolumn{1}{c|}{ Description } \\
\hline orf12-O1 & GTGCGCGACGGGGTGGTA & Reverse for orf12 RT-PCR and quantitative RT-PCR \\
orf13-O1 & CTGCGCTGGCTGCTGGTGTA & Forward for orf13 RT-PCR and quantitative RT-PCR \\
orf13-O1 & CTGCCGCCGGGAGATGC & Reverse for orf13 RT-PCR and quantitative RT-PCR \\
orf14-O1 & CGAACGACGACGAAACG & Forward for orf14 RT-PCR and quantitative RT-PCR \\
orf14-O2 & CGAGCGAGCCGACCATGT & Reverse for orf14 RT-PCR and quantitative RT-PCR \\
ornA-O1 & GATCGACTGGAGATGACC & Forward for ornA RT-PCR and quantitative RT-PCR \\
ornA-O2 & CACGATGTCCACCCCTTC & Reverse for ornA RT-PCR and quantitative RT-PCR \\
pah2-O1 & TCGACGCCGGGGACATCAAT & Forward for pah2 RT-PCR \\
pah2-O2 & CCGCTGGCCGACCTTCTC & Reverse for pah2 RT-PCR \\
pah2-O1 & CCTACGACGGGGGCACCAG & Forward for pah2 quantitative RT-PCR \\
pah2-O2 & TCATGTCGAACGGCGTCAGATTG & Reverse for pah2 quantitative RT-PCR \\
PadpA-O1 & CCCATTGCGACGCTCGCAC & Forward for adpA and promoter region cloning \\
\hline
\end{tabular}

Gene expression analysis by RT-PCR and real-time RT-PCR was performed as previously described by Santamarta et al. (2007). Negative controls to confirm the absence of contaminating DNA on RT-PCR amplification were carried out with each set of primers and Platinum Taq DNA polymerase (Invitrogen). When real-time RTPCR was performed, controls were included using RNA to preclude the amplification of chromosomal DNA. Relative quantification of gene expression was performed by the $2^{-\Delta \Delta C_{t}}$ method.

cDNAs for real-time RT-PCR analysis were synthesized using SuperScript III reverse transcriptase (Invitrogen). In total, $1 \mu \mathrm{g}$ RNA was annealed at $70{ }^{\circ} \mathrm{C}$ for 5 min with 250 pmol random primers (Invitrogen) and $1 \mu 10 \mathrm{mM}$ dNTPs in a final volume of $14 \mu \mathrm{l}$. The mix was then supplemented with $4 \mu \mathrm{l} 5 \times$ First-Strand buffer, $1 \mu \mathrm{l} \mathrm{0.1} \mathrm{M} \mathrm{DTT} \mathrm{and} 1 \mu \mathrm{l}$ SuperScript III reverse transcriptase, and kept at $25{ }^{\circ} \mathrm{C}$ for $5 \mathrm{~min}$ and $55^{\circ} \mathrm{C}$ for $1 \mathrm{~h}$. The retrotranscription reaction was stopped by heating at $70{ }^{\circ} \mathrm{C}$ for $15 \mathrm{~min}$. Real-time RTPCRs were carried out on a StepOnePlus thermocycler (Applied Biosystems). Reactions contained $2 \mu \mathrm{l}$ cDNA reaction mixture diluted $1: 3,10 \mu \mathrm{l}$ SYBR Green PCR Master Mix (Applied Biosystems) and $300 \mathrm{nM}$ specific primers in a volume of $20 \mu \mathrm{l}$, and were performed in triplicate. The $h r d B$-like gene, encoding the major sigma factor in $S$. coelicolor A3(2) (Aigle et al., 2000; Buttner et al., 1990), was used as an internal control to quantify the relative expression of the target genes. PCR conditions were as follows: $2 \mathrm{~min}$ at $50{ }^{\circ} \mathrm{C}, 10 \mathrm{~min}$ at $90{ }^{\circ} \mathrm{C}, 30$ cycles of $15 \mathrm{~s}$ at $95{ }^{\circ} \mathrm{C}$, and $1 \mathrm{~min}$ at $60-64{ }^{\circ} \mathrm{C}$, depending on the primer pair. Specific product amplification was checked by the melting curve and agarose gel electrophoresis. In parallel, control PCRs were performed using RNA as template to preclude amplification of chromosomal DNA. Two biological replicates were employed for each strain; the efficiencies of the primers were measured by serial dilutions of genomic DNA as template.

\section{RESULTS}

\section{Organization of the S. clavuligerus adpA- containing DNA region}

A $5 \mathrm{~kb}$ DNA sequence containing the adpA gene was provided by DSM (Delft, The Netherlands). The DNA sequence and ORFs present in the fragment (Fig. 1a) totally coincide with those later published by the Broad Institute and will be named with the published nomenclature.
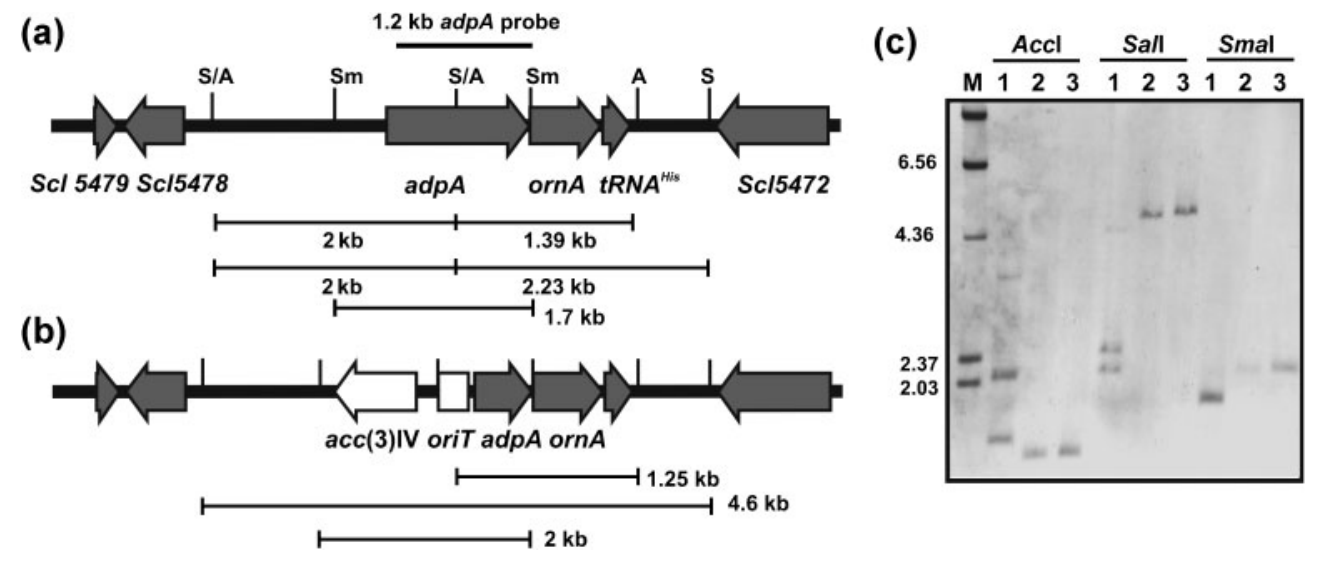

Fig. 1. Organization of the adpA-carrying region in $S$. clavuligerus. (a) Gene organization of a $5 \mathrm{~kb} D N A$ fragment of $S$. clavuligerus carrying the adpA gene. (b) Organization of the same region in S. clavuligerus $\triangle a d p A$ mutants. (c) Pattern of hybridization of Accl-, Sall- and Smal-digested DNA from S. clavuligerus 27064 (1), S. clavuligerus $\Delta a d p A 1$ (2) and S. clavuligerus $\triangle a d p A 2(3)$. 
S. clavuligerus adpA encodes a 399 amino acid protein with $84-88 \%$ identity to those of S. griseus, Streptomyces avermitilis and S. coelicolor. Like other AdpA orthologous proteins, it possesses two helix-turn-helix motifs (amino acids 238-280 and 286-330), characteristic of the AraC/ XylS family of proteins, a domain pfpI/DJ-1 (amino acids 55-193) for dimerization, and a UUA codon-translated leucine residue $\left(\mathrm{Leu}^{223}\right)$.

Upstream, divergent and separated from adpA by a $1.7 \mathrm{~kb}$ non-coding region is SSCG_05478, which encodes a protein of the universal stress family; 83 nt downstream of SSCG_05478 and in the opposite orientation is located a gene encoding a glutamine-D-fructose-6-phosphate amidotransferase. Downstream of $a d p A$ and separated by $10 \mathrm{nt}$ is SSCG_05473, encoding an oligoRNase with $88 \%$ identity to OrnA from S. coelicolor and S. griseus. Next to it, SSCG_100047 encodes a tRNA ${ }^{\text {His }}$, followed 823 nt downstream and in the opposite orientation by an incomplete ORF (SSCG_05472) for a histidine kinase (Fig. 1a). Thus, there is a considerable synteny between this $S$. clavuligerus DNA region and the homologous ones in S. coelicolor and S. griseus.

To understand the effect of AdpA on morphological differentiation and antibiotic production in S. clavuligerus, we proceeded to disrupt the adpA gene by using plasmid $\mathrm{p} \triangle a d p A$. The plasmid was constructed to delete $151 \mathrm{bp}$ of the promoter region and $812 \mathrm{bp}$ of the $5^{\prime}$ end of adpA (Fig. 1b). Plasmid $\mathrm{p} \triangle a d p A$ was transferred to $S$. clavuligerus by conjugation and two of 28 recombinant colonies were apramycin-resistant and kanamycin-sensitive. These exconjugants were analysed by Southern hybridization using a $1.2 \mathrm{~kb}$ DNA probe containing the whole adpA gene. The hybridization pattern obtained (Fig. 1c) is consistent with the deletion of the expected 963 bp region in both exconjugants, which were named $S$. clavuligerus $\triangle a d p A 1$ and $\triangle a d p A 2$.

\section{Morphological differentiation in S. clavuligerus $\triangle a d p A$ mutants depends on culture media}

Growth, aerial mycelium formation and sporulation of $S$. clavuligerus ATCC 27064 and the two $\triangle a d p A$ mutants were studied. In TBO medium, S. clavuligerus ATCC 27064 produced aerial mycelium after 3-4 days of growth, and the characteristic grey-green colour of the spores was observed after 7 days. Only a sparse aerial mycelium was developed by the mutants after 10 days and no spores were formed even after longer incubation times (Fig. 2c). However, in ME medium, an excellent sporulation medium for $S$. clavuligerus ATCC 27064, the $\triangle a d p A$ mutants were able to form aerial mycelium and to sporulate. Since the genetic characterization and morphological behaviour of both exconjugants were identical (data not shown), all the work was performed with exconjugant $\triangle a d p A 1$, named S. clavuligerus $\triangle a d p A$.

\section{Expression of ornA in S. clavuligerus}

The orn $A$ gene, located downstream of and in the same orientation as adpA, is not essential in S. griseus and $S$.
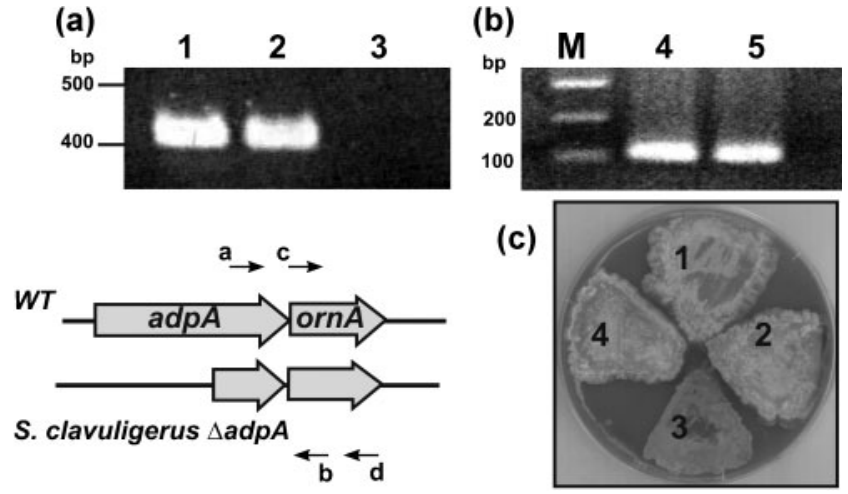

(c)

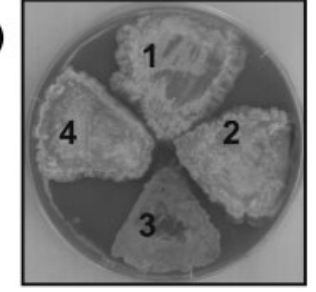

Fig. 2. Characterization of $S$. clavuligerus $\triangle a d p A$. (a) Amplification of the $S$. clavuligerus ATCC 27064 intergenic $\operatorname{adpA-orn} A$ region using oligonucleotides $\operatorname{adpA-ornA-O1}$ and adpA-ornA-O2. Lanes: 1, positive PCR control with DNA as template; 2, RT-PCR amplification of the intergenic region; 3, RTPCR negative control reaction lacking retrotranscriptase. (b) RTPCR amplification of ornA using oligonucleotides ornA-O1 and ornA-O2 with S. clavuligerus ATCC 27064 (lane 4) and S. clavuligerus $\triangle a d p A$ (lane 5). M, molecular mass standard. In the scheme below is shown the location of oligonucleotides $\operatorname{adp} A$ ornA-O1 and adpA-ornA-O2 (marked ' $a$ ' and 'b') and ornA-O1 and ornA-O2 (marked 'c' and 'd'). (c) Growth, aerial mycelium formation and sporulation in TBO medium of $S$. clavuligerus ATCC 27064 (1), S. clavuligerus [pMS83] (2), S. clavuligerus $\triangle a d p A(3)$ and S. clavuligerus $\triangle a d p A$ [pCPA2] (4).

coelicolor, although its deletion partially affects growth and aerial mycelium formation (Ohnishi et al., 2000; Sello \& Buttner, 2008). The small (10 bp) adpA-ornA intergenic region present in $S$. clavuligerus suggests that both genes are transcriptionally coupled in this strain. To assess whether this was the case, oligonucleotides adpA-ornA-O1 and adpA-ornA-O2 were designed to amplify by RT-PCR a $407 \mathrm{bp}$ fragment corresponding to the intergenic region. In addition, oligonucleotides ornA-O1 and ornA-O2 were used to detect ornA expression in $S$. clavuligerus $\triangle a d p A$, in which the promoter and $5^{\prime}$ end of $a d p A$ are deleted. RNA samples were isolated from $24 \mathrm{~h}$ (TSB medium) and $40 \mathrm{~h}$ (SA medium) cultures. The amplification fragment obtained (Fig. 2a, lanes 1 and 2) with oligonucleotides adpA-ornA-O1 and adpA-ornA-O2 confirmed that the two genes are transcriptionally coupled in the wild-type strain. In addition, an amplified fragment corresponding to an ornA transcript was detected in the wild-type and the $\triangle a d p A$ mutant using oligonucleotides ornA-O1 and ornA$\mathrm{O} 2$ (Fig. 2b, lanes 4 and 5); this result confirms the presence of an additional monocistronic ornA mRNA. Real-time RT-PCR, using ornA-O1 and ornA-O2, was performed to quantify the ornA expression level in the wild-type strain and the $\triangle a d p A$ mutant. The relative expression value obtained for ornA in $S$. clavuligerus $\triangle a d p A$ SA cultures grown for $40 \mathrm{~h}$ was 0.148 , which indicates a decrease in expression of 6.7-fold in the mutant compared with the wild-type strain (relative expression value of 1 ). 
This decrease in ornA expression can be explained through the loss of ornA transcripts initiated from the adpA promoter.

\section{Brp specifically binds the adpA promoter region}

Brp is a butyrolactone receptor protein, homologous to $S$. griseus ArpA, and acts as a negative modulator of antibiotic biosynthesis in S. clavuligerus. It recognizes and binds ARE boxes present in the $c c a R$ and brp promoter regions (Santamarta et al., 2005). Bioinformatic analysis and comparison of the adpA promoter region with the $\mathrm{ARE}_{c c a R}$ and $\mathrm{ARE}_{b r p}$ boxes indicated the presence $149 \mathrm{bp}$ upstream of the adpA start codon of a putative ARE sequence, 5' $^{\prime}$ TCTCATGGAGACATAGCGGGGCATGC-3'. This sequence possesses stretches of identity with $S$. clavuligerus $\mathrm{ARE}_{c c a R}$ and $\mathrm{ARE}_{b r p}$, and with the ARE boxes of regulatory gene promoters of other Streptomyces species, including the ArpA-binding sequence in the S. griseus adpA promoter (Fig. 3a).

To determine Brp binding to the adpA promoter region, the electrophoretic mobility of a $448 \mathrm{bp} \mathrm{ARE}_{a d p A^{-}}$-containing fragment in the presence of $S$. clavuligerus $\mathrm{r}$-Brp was tested by EMSAs. In parallel, the binding to the $\mathrm{ARE}_{b r p}$ and

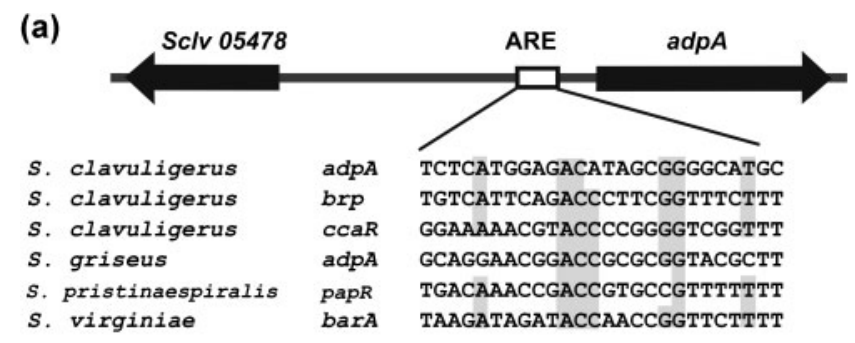

(b)

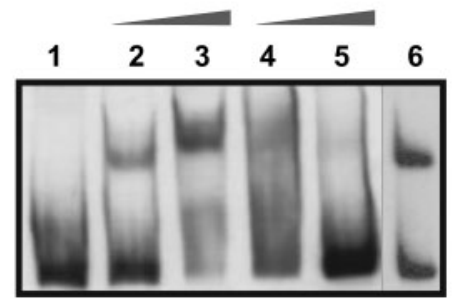

Fig. 3. An ARE box is present upstream of $\operatorname{adp} A$. (a) Location of the ARE box upstream of the adpA gene in $S$. clavuligerus. Sequence of the $\mathrm{ARE}_{a d p A}$ box and comparison with $\mathrm{ARE}_{c c a R}$ and $\mathrm{ARE}_{\text {brp }}$ boxes of $S$. clavuligerus, as well as with ARE sequences present upstream of $S$. griseus adpA, Streptomyces pristinaespiralis papR and Streptomyces virginiae barA. (b) Gel shift electrophoresis of a 448 bp DNA fragment carrying the $A_{R E} E_{a d p A}$ box using pure recombinant $S$. clavuligerus $r$-Brp protein $(0.5 \mu \mathrm{g})$. Lanes: 1 , free probe; 2 and 3, 0.5-4 $\mu \mathrm{g} \mathrm{r-Brp;} 4$ and 5, sequencespecificity assay using one- and 10-fold amounts of unlabelled probe; 6 , sequence-specificity assay using 10-fold amounts of a heterologous unlabelled $445 \mathrm{bp}$ Pvull DNA fragment isolated from pBSKSII.
$\mathrm{ARE}_{\text {ccaR }}$ boxes was tested. A clear mobility shift of the $\mathrm{ARE}_{\text {adp } A^{-} \text {-containing probe was observed using increasing }}$ amounts of r-Brp protein, as shown in Fig. 3(b), lanes 2 and 3. The binding specificity of Brp for this sequence was tested through direct-competition reactions by increasing the amounts of competitor probe, which resulted in a progressively reduced signal of the shifted labelled

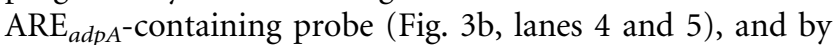
using a heterologous competitor probe that did not disturb the specific binding (Fig. 3b, lane 6). Therefore, the $\mathrm{ARE}_{a d p A}$ sequence is functional and specifically binds Brp.

Real-time RT-PCR quantification of adpA expression was performed in the wild-type strain and the S. clavuligerus $\Delta b r p$ mutant. A consistent slight increase of 2.65 -fold in adpA expression was observed in $S$. clavuligerus $\Delta b r p$ cultures grown for $24 \mathrm{~h}$ in TSB medium. This suggests that Brp acts as a negative modulator of adpA expression in $S$. clavuligerus, as occurs in S. griseus.

\section{The translation of $\operatorname{adpA}$ is regulated by $b / d A$}

The TTA codon-containing adpA gene of $S$. coelicolor is not translated in the S. coelicolor $\Delta$ bldA mutant (Nguyen et al., 2003; Takano et al., 2003). To test whether the same occurs with the TTA codon located in S. clavuligerus adpA, the presence of the AdpA protein was analysed in $S$. clavuligerus ATCC 27064, S. clavuligerus $\Delta a d p A$ and S. clavuligerus $\triangle$ bldA cell extracts through immunodetection assays using anti-AdpA antibodies. Repeatedly, an AdpA inmunodetection signal was observed in $36 \mathrm{~h}$ TSB cell-free extracts of S. clavuligerus ATCC 27064 (Fig. 4a, lane 1), while this band was not present in S. clavuligerus $\triangle a d p A$ or in S. clavuligerus $\triangle$ bldA cell extracts (Fig. 4a, lanes 2 and 3).

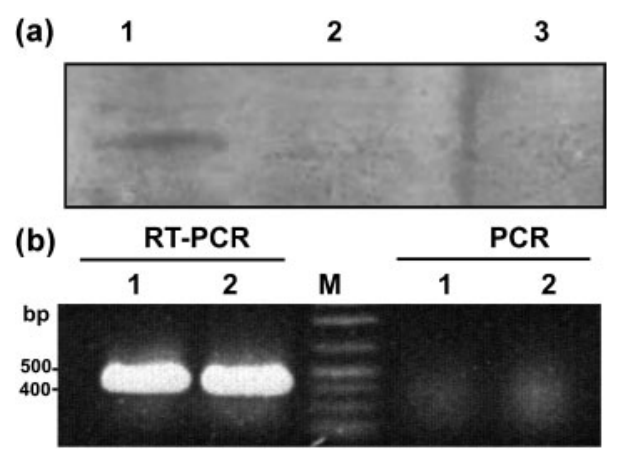

Fig. 4. Immunodetection of AdpA in S. clavuligerus ATCC 27064 and derived mutants. (a) Western blotting with anti-AdpA antibodies of cell extracts ( $5 \mu \mathrm{g}$ protein each) of $S$. clavuligerus ATCC 27064 (1), S. clavuligerus $\triangle a d p A(2)$ and S. clavuligerus $\triangle b / d A$ (3). (b) RT-PCR amplification of adpA (left) and PCR to confirm the absence of contaminating DNA (right). Oligonucleotides $a d p A-\mathrm{O} 3$ and $\operatorname{adpA}-\mathrm{O} 4$ were used on mRNA from $24 \mathrm{~h}$ TSB cultures of S. clavuligerus ATCC 27064 (1) or S. clavuligerus $\triangle b / d A(2)$. 
To confirm that this lack of AdpA protein in the $\Delta b l d A$ mutant was not due to lack of expression, amplification analysis of adpA was performed by RT-PCR. RNA from $24 \mathrm{~h}$ TSB cultures of S. clavuligerus ATCC 27064 and the $\triangle b l d A$ mutant was retrotranscribed using primers adpA-O3 and adpA-O4. The amplification of a DNA fragment corresponding to the adpA transcript in $S$. clavuligerus $\triangle b l d A$ (Fig. $4 \mathrm{~b}$, lanes 1 and 2), confirmed that the absence of AdpA is due to lack of mRNA translation.

\section{Clavulanic acid production is especially affected in S. clavuligerus $\triangle \mathrm{AadpA}$}

Growth and antibiotic production by $S$. clavuligerus ATCC 27064 and S. clavuligerus $\triangle a d p A$ were analysed in defined SA (Fig. 5, upper panels) and in complex TSB media (Fig. 5, lower panels). Inactivation of $a d p A$ did not have a significant effect on growth in either medium (Fig. 5, left panels). Clavulanic acid production was strongly reduced in S. clavuligerus $\Delta a d p$ to $14 \%$ of the wild-type level in TSB medium at $36 \mathrm{~h}$ and to $5 \%$ at $60 \mathrm{~h}$ in SA medium. Production of cephamycin $\mathrm{C}$ by the $\triangle a d p A$ mutant was almost at wild-type level in complex TSB medium but decreased in SA medium after $36 \mathrm{~h}$ of growth (Fig. 5, right panels). Both cephamycin $\mathrm{C}$ and clavulanic acid were restored to control levels in $S$. clavuligerus $\triangle a d p A$ [pCPA2] (Fig. 6a), which carries the $a d p A$ gene in the integrative plasmid pMS83. Introduction of pCPA2 in the S. clavuligerus $\triangle a d p A$ mutant also restored aerial mycelium formation and sporulation in TBO medium (Fig. 2c).

\section{Multiple copies of adpA increase antibiotic production levels in $S$. clavuligerus}

To determine whether antibiotic production was affected by increasing the adpA gene dosage, a DNA fragment containing $a d p A$ with its own promoter was subcloned into the multi-copy plasmid pIJ699, giving plasmid pIJadpA (Table 1). Growth, cephamycin C and clavulanic acid production of the transformant $S$. clavuligerus [pIJadpA] and its control, S. clavuligerus [pIJ699], were analysed in SA-grown cultures. The growth of both transformants was reduced when compared with S. clavuligerus ATCC 27064, probably due to the antibiotic added for plasmid selection. However, production of cephamycin $\mathrm{C}$ and clavulanic acid was clearly enhanced in the strain carrying multiple copies of adpA (Fig. 6b). After $60 \mathrm{~h}$ of culture, clavulanic acid production was of the order of 204 and $218 \%$, respectively, compared with the control S. clavuligerus pIJ699.

\section{Transcriptional analysis of genes involved in clavulanic acid biosynthesis in an $\mathbf{S}$. clavuligerus $\triangle a d p A$ mutant}

Deletion of adpA in $S$. clavuligerus strongly affects clavulanic acid production. To assess whether the pathway-specific regulators of clavulanic acid biosynthesis are under AdpA control, $c c a R$ and claR transcription was analysed by RT-PCR in S. clavuligerus ATCC 27064 and in S. clavuligerus $\triangle a d p A$ (data not shown). Transcriptional studies were performed using as template RNA samples isolated after growth in SA medium for $40 \mathrm{~h}$, at which point the most drastic decrease in clavulanic acid
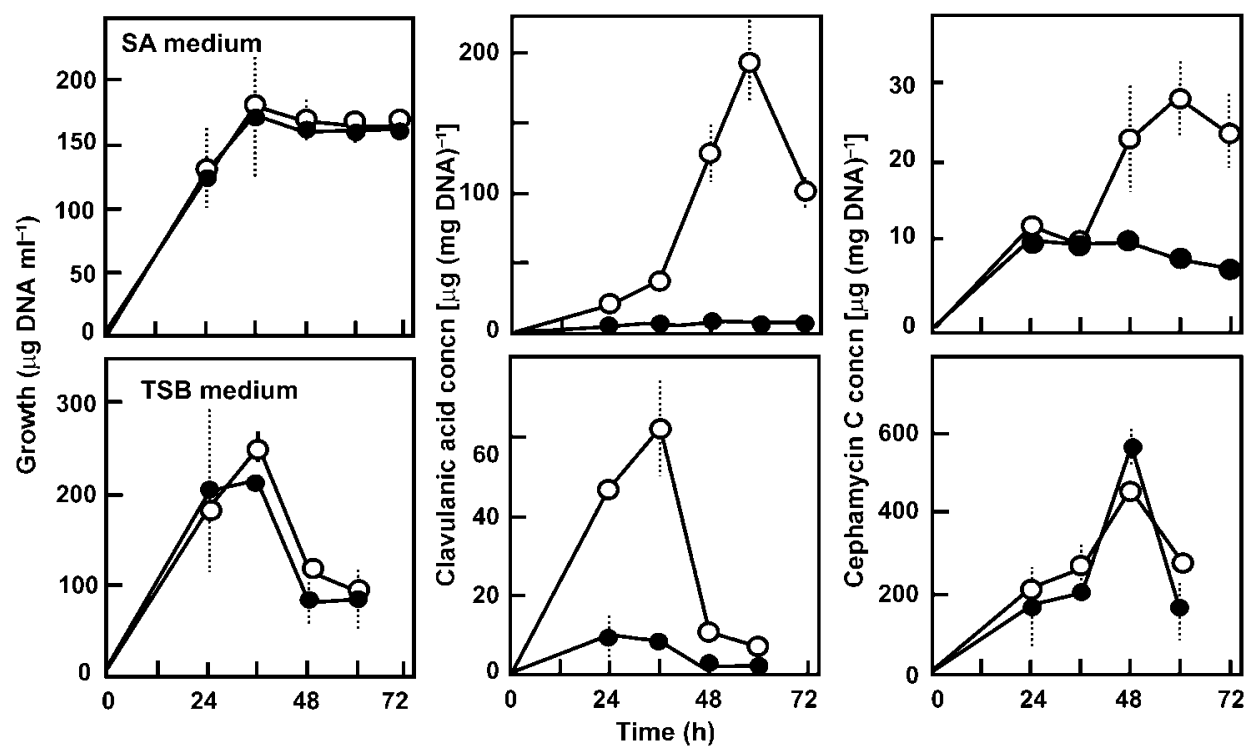

Fig. 5. Cephamycin and clavulanic acid production by $S$. clavuligerus $\Delta a d p A$. Growth (left panels), and production of clavulanic acid (centre panels) and cephamycin C (right panels) by S. clavuligerus ATCC 27064 (open circles) and S. clavuligerus $\triangle a d p A$ (closed circles) grown in SA (upper panels) and TSB (lower panels) media. 


\section{(a)}

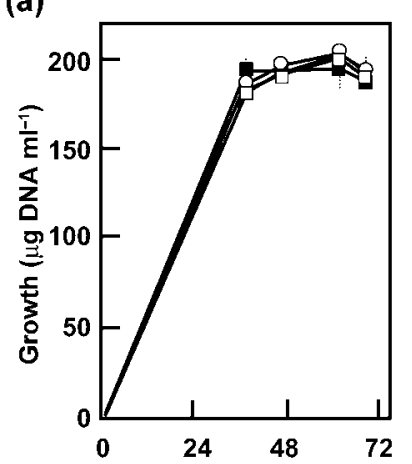

(b)

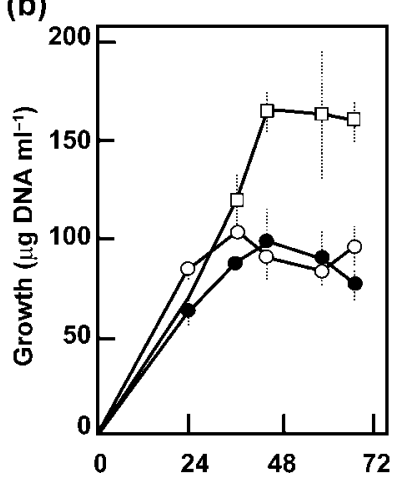

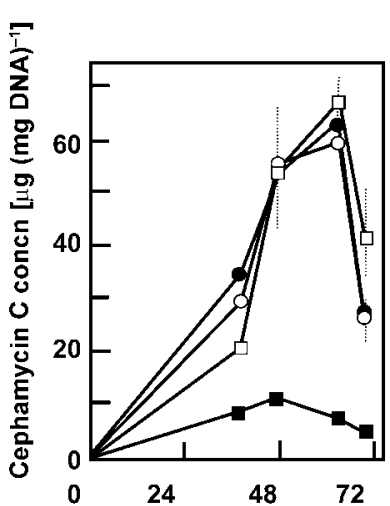

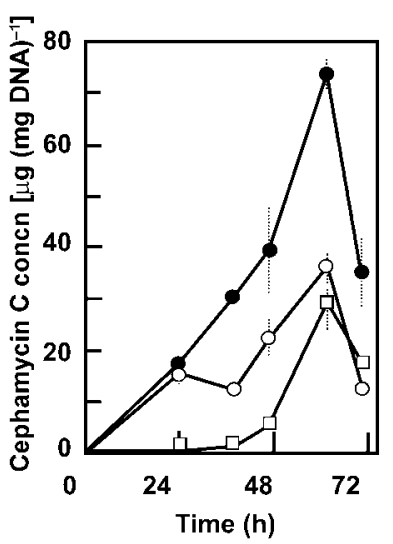

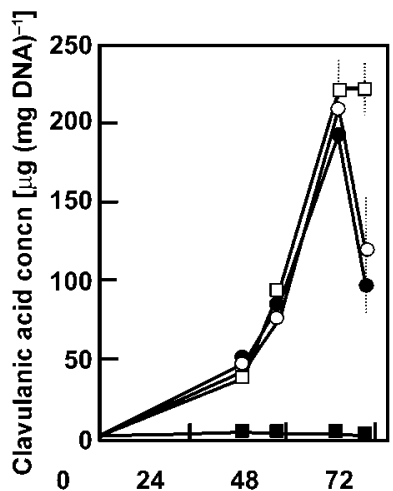

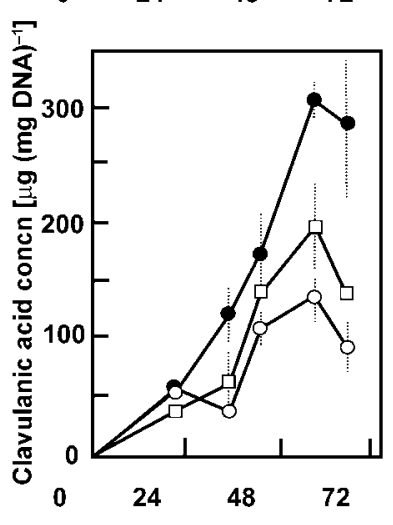

Fig. 6. Complementation of the $\triangle a d p A$ mutant and effect of additional copies of adpA in the control strain. (a) Complementation of $S$. clavuligerus $\triangle a d p A$ by the adpA-carrying pPCA2 integrative plasmid. Growth, and cephamycin C and clavulanic acid production in SA medium of S. clavuligerus 27064 (open squares), S. clavuligerus $\Delta a d p A$ (closed squares), S. clavuligerus [pMS83] (open circles) and S. clavuligerus $\triangle a d p A$ [pCPA2] (closed circles). (b) Effect of multiple copies of $\operatorname{adp} A$ on growth, and cephamycin $\mathrm{C}$ and clavulanic acid production of S. clavuligerus ATCC 27064 (open squares), $S$. clavuligerus [plJ699] (open circles) and S. clavuligerus [plJadpA] (closed circles) grown in SA medium.

production by the $\triangle a d p A$ mutant was observed. No significant differences in amplification of $c c a R$ or $c l a R$ transcripts were observed between the analysed strains (data not shown); therefore, the transcriptional analysis was extended to the whole clavulanic acid cluster. The results indicate that all the genes under study are expressed in the mutant strain, in which clavulanic acid production is not totally abolished. Only the amplification of ceaS2, bls2, pah2, cas2, claR, car and oppA2 transcripts decreased slightly in $S$. clavuligerus $\triangle a d p A$ compared with the wild-type strain. To confirm these differences, a relative quantification by real-time RT-PCR was performed. The expression levels obtained for the different genes in S. clavuligerus adpA in relation to those of the wild-type strain (assigned a relative value of 1) are shown in Fig. 7. Transcription levels of 0.14 and 0.24 were found for $c c a R$ and $c l a R$, encoding positive regulators for clavulanic acid biosynthesis (seven- and fourfold less expression than in the wild-type strain, respectively), while expression of $b r p$ was barely affected, with a relative value of 0.67 (not shown).

All biosynthetic genes analysed appeared to be downregulated in the $\triangle a d p A$ strain. The most dramatic decreases in expression levels were observed in the early biosynthetic genes (ceaS2, bls2, pah2 and cas2), with relative values ranging from 0.0098 (cas2) to 0.052 (pah2). This group of four genes are co-transcribed from the ceaS2 promoter, although cas 2 has been described as also expressed in a monocistronic transcript (Paradkar \& Jensen, 1995). This means a strong decrease in expression of the early genes, of the order of 50-fold lower for ceaS2 and bls2.

The expression level of genes encoding late steps of the pathway (car, gcaS2) in S. clavuligerus $\triangle a d p A$ was variable, since the relative value for car was 0.113 and that for gcaS2 was 0.379 (i.e. about eight- and threefold less than the wild-type strain, respectively). Other essential genes of unknown function in clavulanic acid biosynthesis, such as cyp-fd, orf 12 and orf 13 , showed relative values $(0.132,0.130$ and 0.153 , respectively) similar to those of car. Expression of the oligopeptide permease-encoding gene oppA2 (0.080) was strongly affected, while expression of oppA1, oat 2 and especially orf14 was less affected $(0.215,0.356$ and 0.569 , respectively, i.e. about 4.6-, 3- and 1.7-fold lower). These results allow us to explain the decrease in clavulanic acid production observed in the $\triangle a d p A$ mutant strain and 

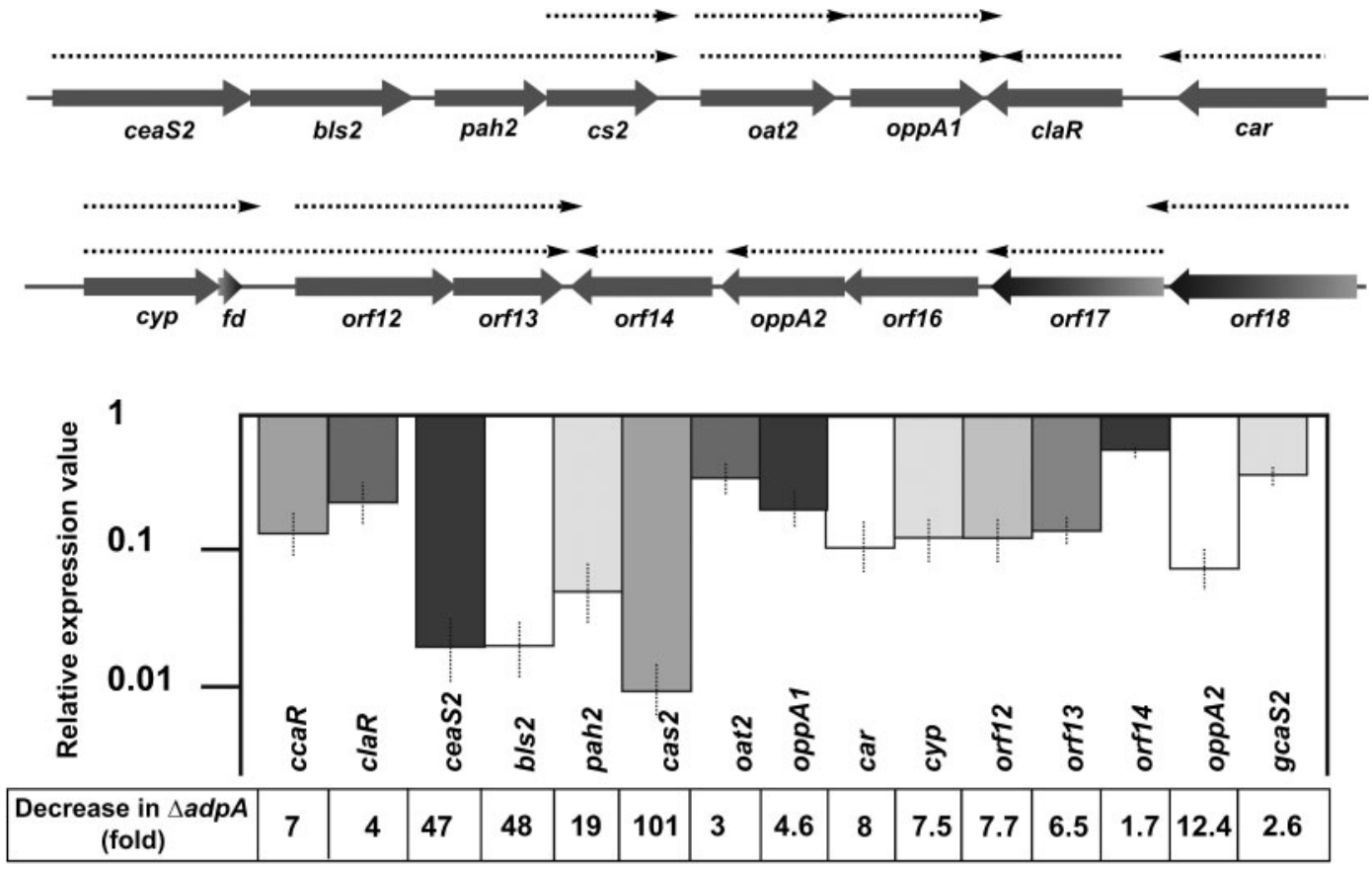

Fig. 7. Expression of clavulanic acid biosynthesis genes. The organization of the S. clavuligerus ATCC 27064 clavulanic acid biosynthesis gene cluster is shown above. Transcriptional units are indicated with arrows. The quantitative RT-PCR of the different genes using the oligonucleotides indicated in Table 2 is shown below. The relative values are referred to 1 , the assigned relative value for the expression of each gene in S. clavuligerus ATCC 27064. Error bars were calculated by measuring the standard deviation among biological replicates of each sample. The mRNA templates were from $40 \mathrm{~h}$ cultures grown in SA medium.

suggests that AdpA acts as a positive regulatory modulator of clavulanic acid gene expression.

\section{DISCUSSION}

Depending on the culture medium, the AdpA-negative mutants of $S$. clavuligerus are blocked in sporulation and show sparse aerial mycelium formation. A similar mediumdependent, sporulation-negative phenotype has been described in S. coelicolor adpA mutants; however, in other Streptomyces species, adpA mutants display a fully bald phenotype (Nguyen et al., 2003; Ohnishi et al., 1999; Pan et al., 2009; Takano et al., 2003). Cephamycin C and especially clavulanic acid formation is impaired in $S$. clavuligerus $\triangle a d p A$. Expression of ornA, encoding an oligoRNase involved in morphological differentiation (Ohnishi et al., 2000; Sello \& Buttner, 2008), is lower in $S$. clavuligerus $\triangle a d p A$. However, the lack of sporulation observed in $S$. clavuligerus $\triangle a d p A$ is not due to the low transcription of ornA, since the strain complemented in trans, S. clavuligerus $\triangle a d p A$ (pCPA2), which still has a low expression of ornA, sporulates normally and produces wild-type levels of cephamycin $\mathrm{C}$ and clavulanic acid.

Since all $a d p A$ genes described so far contain a TTA codon, which is not translated in mutants blocked in the bldA gene, it has been postulated that all the morphological differences observed in $S$. coelicolor bldA mutants are due to lack of $a d p A$ translation (Nguyen et al., 2003; Takano et al., 2003). S. clavuligerus $\Delta$ bldA displays a bald phenotype but produces both cephamycin and clavulanic acid; therefore, the $S$. clavuligerus $\triangle a d p A$ and $S$. clavuligerus $\triangle$ bldA mutants exhibit different phenotypes in relation to aerial mycelium formation and antibiotic production. This might be due to the expression or lack of expression of other still-uncharacterized genes; furthermore, $S$. clavuligerus $\triangle$ bldA correctly translates the TTA codoncontaining $c c a R$ gene, for the clavulanic acid/cephamycin C-specific regulatory protein CcaR (Trepanier et al., 2002; Santamarta, 2002).

The different behaviour of the $S$. clavuligerus bldA mutant with respect to $a d p A$ and $c c a R$ translation might be due to the differences in the TTA $5^{\prime}$ flanking nucleotides (Trepanier et al., 2002). After comparison of all Streptomyces bldA-dependent TTA codons it has been suggested that TTAY sequences (where $\mathrm{Y}$ is $\mathrm{C}$ or $\mathrm{T}$ ) are susceptible to be bldA-dependent as is the case for the TTA codon in $\operatorname{adpA}$ (TTAC), while TTAR sequences (where $\mathrm{R}$ is $\mathrm{G}$ or A), such as in the TTA codon of $c c a R$ (TTAG), are not bldA-dependent. As shown above, the absence of AdpA detection by immunoassays of $S$. clavuligerus $\Delta b l d A$ supports the theory of Trepanier and co-workers. 
The decrease of cephamycin C and, especially, of clavulanic acid production in $S$. clavuligerus $\triangle a d p A$ and their overproduction in transformants carrying multiple copies of adpA suggest that AdpA is a positive modulator in the antibiotic regulatory cascade of $S$. clavuligerus. However, the observed effect is more drastic in relation to clavulanic acid production, probably reflecting differences in the regulatory cascades for the two antibiotics (Paradkar \& Jensen, 1998). The transcription in the $\Delta a d p A$ mutant of clavulanic acid biosynthesis regulatory genes $c c a R$ and $c l a R$ was about seven- and fourfold lower than in the wild-type strain, which explains the strong decrease in expression of genes ceaS2, bls2, pah2 and cas2 for the early steps of the clavulanic acid pathway as well as the moderate decrease of the late biosynthesis genes.

Direct AdpA binding to sequences in the pathway-specific regulatory genes that control antibiotic production has been demonstrated in S. griseus, S. ansochromogenes and S. coelicolor (Higashi et al., 2007; Pan et al., 2009; Park et al., 2009; Tomono et al., 2005a). The consensus sequence in $S$. griseus is $5^{\prime}$-TGGCSNGWWY-3', and two types of AdpA binding have been described. In type I, the binding site contains two consensus sequences, while in type II, AdpA binds a single consensus sequence. In the intergenic $\mathrm{cmcH}-$ $c c a R$ region, between the ARE box and the tsp points described for $c c a R$, two possible sequences for AdpA binding are located: (i) $406 \mathrm{bp}$ from the ATG start codon there is a single (type II) sequence, $5^{\prime}$-TGGCCGGATT-3'; and (ii) $565 \mathrm{nt}$ upstream from the ATG there are two direct sequences, 3'-TGGCCCTTTT-14-TGGCCGCTGT-5'. In both cases, the sequences are located in the DNA strand complementary to $c c a R$. Whether these sequences are true sites for AdpA binding has not yet been confirmed, since the purification of $S$. clavuligerus recombinant AdpA has been hampered by the instability in $E$. coli of all the expression vectors carrying adpA that have been constructed.

The butyrolactone receptor Brp binds to the ARE boxcontaining probe, as shown by EMSA; in addition, it has been reported that a Brp-disrupted strain produces 1.5- to threefold more clavulanic acid and cephamycin $C$ than the wild-type strain (Santamarta et al., 2005). This work demonstrates a connection between the butyrolactone and AdpA regulation systems: Brp binds an ARE box present upstream of $a d p A$, which leads to repression of $a d p A$ in the wild-type strain and to a 2.5 -fold increase of adpA transcript in the $S$. clavuligerus Brp-disrupted mutant. The AdpA regulation pattern shown by $S$. clavuligerus resembles that described for S. griseus (Ohnishi et al., 1999, 2005). Thus, ccaR expression is controlled directly by $\operatorname{Brp}$ and indirectly through the Brp-dependent AdpA regulator.

\section{ACKNOWLEDGEMENTS}

This work was supported by Grants of the Spanish Ministry of Science and Technology (BIO2006-14853), the Junta de Castilla y León (GR117) and the European Community (Actinogen LSHMCT-2004-
005224). M. T. L.-G. received a fellowship from the Junta de Castilla y León. We appreciate the $S$. clavuligerus $\Delta b l d A$ strain, received from Drs B. Leskiw and S. E. Jensen (Department of Biological Science, University of Alberta, Canada), DNA sequences obtained from Dr Wilbert Heijne (DSM, The Netherlands) and plasmid pMS83 obtained from Dr Maggie Smith (University of Aberdeen, UK)

\section{REFERENCES}

Aigle, B., Wietzorrek, A., Takano, E. \& Bibb, M. J. (2000). A single amino acid substitution in region 1.2 of the principal sigma factor of Streptomyces coelicolor A3(2) results in pleiotropic loss of antibiotic production. Mol Microbiol 37, 995-1004.

Burton, K. (1968). Determination of DNA concentration with diphenylamine. Methods Enzymol 12, 163-166.

Buttner, M. J., Chater, K. F. \& Bibb, M. J. (1990). Cloning, disruption, and transcriptional analysis of three RNA polymerase sigma factor genes of Streptomyces coelicolor A3(2). J Bacteriol 172, 3367-3378.

Chater, K. F. \& Chandra, G. (2008). The use of the rare UUA codon to define "expression space" for genes involved in secondary metabolism, development and environmental adaptation in Streptomyces. J Microbiol 46, 1-11.

Fernández-Moreno, M. A., Caballero, J. L., Hopwood, D. A. \& Malpartida, F. (1991). The act cluster contains regulatory and antibiotic export genes, direct targets for translational control by the bldA tRNA gene of Streptomyces. Cell 66, 769-780.

Gregory, M. A., Till, R. \& Smith, M. C. (2003). Integration site for Streptomyces phage $\Phi_{B} \mathrm{~T}_{1}$ and development of site-specific integration vectors. J Bacteriol 185, 5320-5323.

Gust, B., Challis, G. L., Fowler, K., Kieser, T. \& Chater, K. F. (2003). PCR-targeted Streptomyces gene replacement identifies a protein domain needed for biosynthesis of the sesquiterpene soil odor geosmin. Proc Natl Acad Sci U S A 100, 1541-1546.

Higashi, T., Iwasaki, Y., Ohnishi, Y. \& Horinouchi, S. (2007). A-factor and phosphate depletion signals are transmitted to the grixazone biosynthesis genes via the pathway-specific transcriptional activator GriR. J Bacteriol 189, 3515-3524.

Higgens, C. E., Hamill, R. L., Sands, T. H., Hoehn, M. M., Davis, N. E., Nagarajan, R. \& Boeck, L. D. (1974). The occurrence of deacetylcephalosporin C in fungi and Streptomyces. J Antibiot 27, 298-300.

Kato, J. Y., Suzuki, A., Yamazaki, H., Ohnishi, Y. \& Horinouchi, S. (2002). Control by A-factor of a metalloendopeptidase gene involved in aerial mycelium formation in Streptomyces griseus. J Bacteriol 184, 6016-6025.

Kato, J. Y., Chi, W. J., Ohnishi, Y., Hong, S. K. \& Horinouchi, S. (2005). Transcriptional control by A-factor of two trypsin genes in Streptomyces griseus. J Bacteriol 187, 286-295.

Kieser, T. \& Melton, R. E. (1988). Plasmid pIJ699, a multi-copy positive-selection vector for Streptomyces. Gene 65, 83-91.

Kieser, T., Bibb, M. J., Buttner, M. J., Chater, K. F. \& Hopwood, D. A. (2000). Practical Streptomyces Genetics. Norwich, UK: John Innes Foundation.

Lawlor, E. J., Baylis, H. A. \& Chater, K. F. (1987). Pleiotropic morphological and antibiotic deficiencies result from mutations in a gene encoding a tRNA-like product in Streptomyces coelicolor A3(2). Genes Dev 1, 1305-1310.

Liras, P. \& Martín, J. F. (2005). Assay methods for detection and quantification of antimicrobial metabolites produced by Streptomyces clavuligerus. In Methods in Biotechnology, vol. 18, pp. 149-163. Edited by J. L. Barredo. Totowa, NJ: Humana Press. 
Lorenzana, L. M., Pérez-Redondo, R., Santamarta, I., Martín, J. F. \& Liras, P. (2004). Two oligopeptide-permease-encoding genes in the clavulanic acid cluster of Streptomyces clavuligerus are essential for production of the $\beta$-lactamase inhibitor. J Bacteriol 186, 3431-3438.

Nguyen, K. T., Tenor, J., Stettler, H., Nguyen, L. T., Nguyen, L. D. \& Thompson, C. J. (2003). Colonial differentiation in Streptomyces coelicolor depends on translation of a specific codon within the adpA gene. J Bacteriol 185, 7291-7296.

Ohnishi, Y., Kameyama, S., Osaka, H. \& Horinouchi, S. (1999). The A-factor regulatory cascade leading to streptomycin biosynthesis in Streptomyces griseus: identification of a target gene of the A-factor receptor. Mol Microbiol 34, 102-111.

Ohnishi, Y., Nishiyama, Y., Sato, R., Kameyama, S. \& Horinouchi, S. (2000). An oligoribonuclease gene in Streptomyces griseus. J Bacteriol 182, 4647-4653.

Ohnishi, Y., Yamazaki, H., Kato, J. Y., Tomono, A. \& Horinouchi, S. (2005). AdpA, a central transcriptional regulator in the A-factor regulatory cascade that leads to morphological development and secondary metabolism in Streptomyces griseus. Biosci Biotechnol Biochem 69, 431-439.

Pan, Y., Liu, G., Yang, H., Tian, Y. \& Tan, H. (2009). The pleiotropic regulator AdpA-L directly controls the pathway specific activator of nikkomycin biosynthesis in Streptomyces ansochromogenes. Mol Microbiol 72, 710-723.

Paradkar, A. S. \& Jensen, S. E. (1995). Functional analysis of the gene encoding the clavaminate synthase 2 isoenzyme involved in clavulanic acid biosynthesis in Streptomyces clavuligerus. J Bacteriol 177, 13071314.

Paradkar, A. S. \& Jensen, S. E. (1998). A pathway-specific transcriptional activator regulates late steps of clavulanic acid biosynthesis in Streptomyces clavuligerus. Mol Microbiol 27, 831-843.

Park, S. S., Yang, Y. H., Song, E., Kim, E. J., Kim, W. S., Sohng, J. K., Lee, H. C., Liou, K. K. \& Kim, B. G. (2009). Mass spectrometric screening of transcriptional regulators involved in antibiotic biosynthesis in Streptomyces coelicolor A3(2). J Ind Microbiol Biotechnol 36, 1073-1083.

Pérez-Llarena, F. J., Liras, P., Rodríguez-García, A. \& Martín, J. F. (1997). A regulatory gene $(c c a R)$ required for cephamycin and clavulanic acid production in Streptomyces clavuligerus: amplification results in overproduction of both $\beta$-lactam compounds. J Bacteriol 179, 2053-2059.

Pérez-Redondo, R., Rodríguez-Garcia, A., Martín, J. F. \& Liras, P. (1999). Deletion of the $p y c$ gene blocks clavulanic acid biosynthesis except in glycerol-containing medium: evidence for two different genes in formation of the C3 unit. J Bacteriol 181, 6922-6928.

Rodríguez-García, A., Santamarta, I., Pérez-Redondo, R., Martín, J. F. \& Liras, P. (2006). Characterization of a two-gene operon epeRA involved in multidrug resistance in Streptomyces clavuligerus. Res Microbiol 157, 559-568.

Romero, J., Liras, P. \& Martín, J. F. (1984). Dissociation of cephamycin and clavulanic acid biosynthesis in Streptomyces clavuligerus. Appl Microbiol Biotechnol 20, 318-325.
Sambrook, J., Fritsch, E. F. \& Maniatis, T. (1989). Molecular Cloning: a Laboratory Manual, 2nd edn. Cold Spring Harbor, NY: Cold Spring Harbor Laboratory.

Sánchez, L. \& Braña, A. F. (1996). Cell density influences antibiotic biosynthesis in Streptomyces clavuligerus. Microbiology 142, 12091220.

Santamarta, I. (2002). Control de la expresión de los genes de biosíntesis de cefamicina C en Streptomyces clavuligerus por la proteína CcaR. $\mathrm{PhD}$ thesis, Universidad de León (León).

Santamarta, I., Pérez-Redondo, R., Lorenzana, L. M., Martín, J. F. \& Liras, P. (2005). Different proteins bind to the butyrolactone receptor protein ARE sequence located upstream of the regulatory $c c a R$ gene of Streptomyces clavuligerus. Mol Microbiol 56, 824-835.

Santamarta, I., López-Garcia, M. T., Pérez-Redondo, R., Koekman, B., Martín, J. F. \& Liras, P. (2007). Connecting primary and secondary metabolism: AreB, an IclR-like protein, binds the AREccaR sequence of $S$. clavuligerus and modulates leucine biosynthesis and cephamycin $\mathrm{C}$ and clavulanic acid production. Mol Microbiol 66, 511-524.

Sello, J. K. \& Buttner, M. J. (2008). The oligoribonuclease gene in Streptomyces coelicolor is not transcriptionally or translationally coupled to adpA, a key BldA target. FEMS Microbiol Lett 286, 60-65.

Takano, E., Tao, M., Long, F., Bibb, M. J., Wang, L., Li, W., Buttner, M. J., Bibb, M. J., Deng, Z. X. \& Chater, K. F. (2003). A rare leucine codon in adpA is implicated in the morphological defect of bldA mutants of Streptomyces coelicolor. Mol Microbiol 50, 475-486.

Tomono, A., Tsai, Y., Yamazaki, H., Ohnishi, Y. \& Horinouchi, S. (2005a). Transcriptional control by A-factor of $s t r R$, the pathwayspecific transcriptional activator for streptomycin biosynthesis in Streptomyces griseus. J Bacteriol 187, 5595-5604.

Tomono, A., Tsai, Y., Ohnishi, Y. \& Horinouchi, S. (2005b). Three chymotrypsin genes are members of the AdpA regulon in the A-factor regulatory cascade in Streptomyces griseus. J Bacteriol 187, 6341-6353.

Trepanier, N. K., Jensen, S. E., Alexander, D. C. \& Leskiw, B. K. (2002). The positive activator of cephamycin C and clavulanic acid production in Streptomyces clavuligerus is mistranslated in a bldA mutant. Microbiology 148, 643-656.

White, J. \& Bibb, M. (1997). bldA dependence of undecylprodigiosin production in Streptomyces coelicolor A3(2) involves a pathwayspecific regulatory cascade. J Bacteriol 179, 627-633.

Yamazaki, H., Ohnishi, Y. \& Horinouchi, S. (2000). An A-factordependent extracytoplasmic function sigma factor ( $\left.\sigma^{\text {AdsA }}\right)$ that is essential for morphological development in Streptomyces griseus. J Bacteriol 182, 4596-4605.

Yamazaki, H., Ohnishi, Y. \& Horinouchi, S. (2003). Transcriptional switch on of $s s g A$ by A-factor, which is essential for spore septum formation in Streptomyces griseus. J Bacteriol 185, 1273-1283.

Yamazaki, H., Tomono, A., Ohnishi, Y. \& Horinouchi, S. (2004). DNA-binding specificity of AdpA, a transcriptional activator in the Afactor regulatory cascade in Streptomyces griseus. Mol Microbiol 53, $555-572$.

Edited by: C. W. Chen 